\title{
Corneal Elevation Asymmetry Vector as a Predictor of One-year-averaged Orthokeratology Lens Decentration in Low Astigmats
}

Qiong Xu

Peking University People's Hospital

Kai Wang ( $\nabla$ wang_kai@263.net)

Peking University People's Hospital

Yan Li

Peking University People's Hospital

Jia Qu

Peking University People's Hospital

Mingwei Zhao

Peking University People's Hospital

\section{Research Article}

Keywords: Myopia, Orthokeratology, Lens Decentration, Corneal Elevation Asymmetry Vector

Posted Date: September 8th, 2021

DOl: https://doi.org/10.21203/rs.3.rs-535008/v1

License: (a) (i) This work is licensed under a Creative Commons Attribution 4.0 International License. Read Full License 


\section{Abstract}

Significance: This study establishes a novel and practical method for locating orthokeratology lens decentration. The general lens decentration over a one-year period could be partially predicted using the corneal elevation asymmetry vector.

Purpose: The predictive ability of the pretreatment corneal elevation asymmetry vector for the average orthokeratology lens decentration in Chinese myopic adolescents with low astigmatism was explored.

Methods: Thirty-five myopic participants (35 eyes) were enrolled in this prospective study. The follow-up time was up to one year. Various pretreatment corneal parameters were analyzed to identify potential candidates for lens decentration prediction. An accurate and objective method was developed in MATLAB to calculate the corneal asymmetry vector, which was defined as the sum of the elevation vector at the boundary between the reverse curve and the alignment curve. The program also locates a precise lens decentration vector. The relationship between pretreatment corneal parameters and lens decentration was analyzed using linear regression.

Results: Lens decentration stabilized after 1 month. The averaged lens decentration vector was calculated to indicate the general decentration situation after stabilization. The magnitude of the averaged decentration varied from $0.07 \mathrm{~mm}$ to $1.35 \mathrm{~mm}$, with $91 \%$ measuring less than $1.0 \mathrm{~mm}$. Inferotemporal decentration was the most common type (66\%). Among all the pretreatment parameters, only the corneal asymmetry vector significantly correlated with the averaged lens decentration in horizontal coordinates $(R=0.374, p=.027)$. The vertical asymmetry vector was close to significance when correlated with the vertical averaged lens decentration vector $(R=0.327, p=.055)$. The angle of the corneal elevation asymmetry vector significantly contributed to the averaged lens decentration angle $(R=0.344$, $\mathrm{p}=.043)$.

Conclusions: The association between pretreatment corneal elevation asymmetry vector and one-yearaveraged orthokeratology lens decentration may assist in fitting and understanding the performance of orthokeratology lenses.

\section{Introduction}

Studies have consistently reported that orthokeratology treatment significantly retards myopia progression in children by slowing ocular growth. ${ }^{1-5}$ Orthokeratology lenses are reverse-geometry rigid gas-permeable contact lenses that temporarily reshape the cornea during sleep. ${ }^{6}$ The central cornea is flattened to correct central myopic refractive errors during sleep with the lenses. The paracentral cornea is relatively steepened by the orthokeratology lens. This induces peripheral myopic defocus, which may be a mechanism of myopia control. ${ }^{2,7}$ The flattened central cornea is referred to as the treatment zone. The orthokeratology lens fitting conditions can be evaluated with corneal topography after treatment, and a 
"bullseye" pattern in which the treatment zone covers the pupillary area and is surrounded by a positive defocus ring is considered ideal. ${ }^{3,4}$

If the treatment zone is decentered, the paracentral defocus ring may fall into the pupillary area, resulting in reduced visual performance due to the induction of unwanted astigmatism and higher-order aberrations. ${ }^{8-10}$ Severe lens decentration will not only reduce the optical quality but also lead to complications in both the structure and function of the cornea since ortho-k lenses closely contact the cornea overnight. Patients may suffer from corneal epithelial damage, corneal central islands, indentation, etc. and may complain of ghosting, double vision, glare, halos and foreign body sensations. Under these conditions, lenses should not be worn, and the lens fitting should be reassessed. ${ }^{10,11}$

Some modified manual methods have been introduced to quantify lens decentration from corneal topography maps. Several points surrounding the central flattened area in which the power is all zero on the difference topography map were plotted manually. Then these points were used to fit a circle, whose center was defined as the center of the treatment zone. ${ }^{12-14}$ However, these methods are subjective, and their repeatability is poor. Prior to investigating factors that can cause lens decentration, it would be useful to develop a more precise method by which to quantify lens decentration. Li et al. ${ }^{15}$ found that lens decentration changed with time and did not stabilize until six months after orthokeratology treatment and that a longer follow-up period was needed.

The exact cause of lens decentration and a reliable strategy for predicting and preventing lens decentration have not yet been addressed. Researchers have found that a higher corneal astigmatism or higher sagittal height difference for an 8.00-mm chord leads to a larger lens decentration. ${ }^{13,16,17}$ Therefore, it is suggested that patients with corneal astigmatism of less than $1.50 \mathrm{D}$ (with-the-rule) or a sagittal height difference over $20 \mu \mathrm{m}$ should receive a spherically designed orthokeratology lens. 2, 5, 7, 16 In patients with a higher corneal astigmatism, toric orthokeratology lenses have been developed to reduce lens decentration. However, lens decentration is commonly observed clinically, despite a successful lensfitting process in patients with low corneal astigmatism. ${ }^{14,18}$ In addition to corneal astigmatism and the sagittal height difference, the sum of the corneal asphericity (Q-value) differences between the nasaltemporal and superior-inferior quadrants is considered to be a sensitive predictor of lens decentration. Higher corneal Q-value differences indicated a greater possibility of lens decentration. In addition, asymmetry of the corneal elevation between different quadrants has been investigated for predicting the magnitude and direction of lens decentration. ${ }^{14}$ However, to the best of our knowledge, no study to date has used objective methods to predict lens decentration over one year of orthokeratology lens wearing.

This study was performed to develop a more reliable and objective method by which to locate orthokeratology lens decentration. The one-year-averaged lens decentration was investigated as a novel convenient indicator to represent the overall lens decentration position through the whole year. It was calculated by vector synthesis using decentration data from every follow-up visit after the first month 
during a one-year period. In addition, a simplified objective indicator-the corneal elevation asymmetry vector-was calculated for lens decentration prediction in the clinic.

\section{Subjects And Methods Study Subjects}

This prospective study was conducted from April 2017 to December 2018 at the Optometry Center of Peking University People's Hospital. Informed consent was obtained from each participant or their guardians before their participation in this study.

The inclusion criteria were as follows: age between 8 and 18 years, myopic spherical refractive error between -1.0 and $-4.0 \mathrm{D}$, refractive astigmatism up to $-1.5 \mathrm{D}$, visual acuity correctable to $20 / 20$ or better, and no contact lens-related contraindications. None of the participants had previously received orthokeratology treatment. All procedures performed in this study were in accordance with the ethical standards of the Institutional Research Ethics Committee of Peking University People's Hospital and with the 1964 Helsinki declaration and its later amendments.

In all, 35 participants were included in this study. They underwent orthokeratology treatment for one year and participated in regular follow-up visits.

\section{Corneal Topography}

A corneal topographer (Sirius, CSO, Italy) was utilized to capture the corneal topography at baseline and during all measurement sessions for all participants. Three repeated measurements were carried out, and the best-focused image (with an accuracy over 95\%) was used. Several pretreatment corneal parameters were directly obtained from the topography profile, including the flat and steep $\mathrm{K}$ readings, corneal astigmatism, corneal asphericity (Q-value) values (including the mean and four quadrant values), and horizontal visible iris diameter.

\section{Pretreatment corneal elevation asymmetry vector-- Calculated according to the anterior elevation map}

The asymmetry of the cornea at the chord of $7.2 \mathrm{~mm}$, which lies at the boundary between the reverse curve and the alignment curve, was considered to be important in lens centration (Figure. 1A). ${ }^{19}$ To calculate the overall sagittal height at this boundary corresponding to the cornea at all 256 semimeridians (Figure. 1B), we decomposed the sagittal height into $\mathrm{Sag}_{\mathrm{x}}$ and $\mathrm{Sag}_{\mathrm{y}}$ by a vector-based method $\left(\operatorname{Sag}_{\mathrm{x}}=\mathrm{Sag}^{\star} \operatorname{Cosa}, \mathrm{Sag}_{\mathrm{y}}=\mathrm{Sag}^{\star} \operatorname{sina}\right.$ ) (Figure. 1C). Then, another customized MATLAB (version 7.0; MathWorks Corporation, Natick, Massachusetts, USA) program was used to calculate the sum of the sagittal height vectors in both the $\mathrm{x}$ and $\mathrm{y}$ directions. For lens centration, the sagittal height asymmetry 
represents the power to tilt, which is defined as the corneal elevation asymmetry vector. The twodimensional corneal elevation asymmetry vectors were summed using the following equations:

$$
\begin{aligned}
& V_{x}=\sum_{i=1}^{256} \operatorname{Sag}_{i} \cdot \cos \left(\alpha_{i}\right) \\
& V_{y}=\sum_{i=1}^{256} \operatorname{Sag}_{i} \cdot \sin \left(\alpha_{i}\right)
\end{aligned}
$$

Eq. (2)

where $\mathrm{Sag}_{i}$ and $a_{i}$ are the sagittal height and angle at each semimeridian, respectively. $\mathrm{V}_{\mathrm{x}}$ represents the corneal elevation asymmetry vector at the horizontal axis, and $V_{y}$ represents the corneal elevation asymmetry vector at the vertical axis. The original point of this coordinate system is the corneal vertex. The calculation was conducted by two experienced, independent researchers.

\section{Orthokeratology Lens Fitting}

The orthokeratology contact lenses used in this study were four-zone reverse-geometry gas-permeable rigid contact lenses (Euclid, Herndon, VA, USA) composed of oprifocon A (Boston Equalens II; Bausch \& Lomb, Rochester, NY) (DK: $85^{\star} 10^{-11}\left[\mathrm{~cm}^{2} / \mathrm{s}\right]\left[\mathrm{mL} \mathrm{O}_{2} / \mathrm{mL}^{\star} \mathrm{mm} \mathrm{Hg}\right]$ ). The lens was fitted using the conventional fitting method. The first trial lenses were determined by the same experienced clinician based on the horizontal visible iris diameter, the simulated corneal curvature of the flattest meridian, the corneal eccentricity and anterior corneal sagittal height difference over an 8-mm chord.

The lens fitting suitability was evaluated using static and dynamic corneal fluorescein pattern and topography map collected after a 30-minute eye-closure trial. The desired lenses were ordered based on the fluorescein evaluation, the post-trial wear topography map and the over-refraction result.

After lens delivery, all participants were asked to wear their orthokeratology lenses for at least 8 hours every night during this study. The routine follow-up visits were scheduled at 1 day, 1 week, 1 month, 3 months, 6 months, 9 months and 12 months. Each visit included compliance with lens wearing and lens care procedures, unaided visual acuity measurements, corneal topography and slit-lamp examinations. Data at 1 week, 1 month, 3 months, 6 months, 9 months and 12 months was collected for analysis.

\section{Location of Orthokeratology Lens Decentration}


The results were analyzed by two experienced, independent observers. To determine the lens decentration from the corneal topography maps, the tangential curvature of the cornea of each participant before and after orthokeratology treatment at each visit was exported from the Sirius system as a $31 * 256$ matrix, and a tangential power difference map was calculated by a customized MATLAB program. Then this program automatically selected the turning point from minus to plus on the power difference map. These 256 points composed the boundary of the treatment zone. Then, a leastsquares method in the MATLAB program was used to determine the best-fit circle of these boundary points, and the center of the circle was identified as the treatment zone center (Figure. 1D). ${ }^{14}$

The vector from the center of the circle to the corneal vertex was defined as the Type I lens decentration (illustrated on both the $\mathrm{x}$ - and $\mathrm{y}$-axes, with the original point of this coordinate system at the corneal vertex, where $\mathrm{H}$ represents the center of the treatment zone relative to the corneal vertex in the horizontal direction and $\mathrm{V}$ represents the center of the treatment zone relative to the corneal vertex in the vertical direction). The vector from the circle center to the pupil center was defined as the Type II lens decentration. The lens decentration magnitude was recorded as the absolute value, which could be calculated using the following equation.

\section{Lens decentration magnitude $=\sqrt{H^{2}+V^{2}}$}

The lens decentration vector could also represent the decentration direction, i.e., an $\mathrm{H}$ below 0 on the topography map depicted the lens decentered to the temporal side of the right eye, while an $\mathrm{H}$ above 0 indicated the lens decentered to the nasal side of the right eye, a $\mathrm{V}$ below 0 indicated the lens decentered to the inferior side, and a $\mathrm{V}$ above 0 indicated the lens decentered to the superior side.

Both the lens decentration angle and the corneal asymmetry vector angle were recorded using absolute degrees, which could be calculated using the following equation.

\section{Lens decentration angle $=\frac{180^{\circ}}{\pi} * \tan ^{-1}(H / V)$}

The $0^{\circ}$ semimeridian starts from 3 o'clock on the topography map and moves counterclockwise to the $360^{\circ}$ semimeridian (back to 3 o'clock).

\section{Statistical Analysis}

The lens decentration data were analyzed using the SPSS software package (version 23.0; IBM Corporation, Armonk, NY). Data from the right eyes were used for analysis. The baseline corneal and lens 
decentration parameters at different visits were first tested for normality using the Shapiro-Wilk test. Differences in Type I and Type II lens decentration were compared using paired-sample t-tests. Lens decentration parameters over different visits were analyzed by a repeated-measures analysis of variance (RM-ANOVA), with post hoc tests. The baseline parameters, including the $\mathrm{Vx}, \mathrm{Vy}, \mathrm{K}$ readings, corneal astigmatism, Q values, horizontal visible iris diameter, and spherical equivalent refraction, were analyzed against the averaged lens decentration coordinates using linear regression analysis, respectively. The intraclass correlation coefficient (ICC), coefficient of repeatability (COR), and standard deviation (SD) were used to evaluate the repeatability and reproducibility of the experiment. ${ }^{20,21} \mathrm{~A}$ critical $p$ value of 0.05 was used to represent statistical significance.

\section{Results}

\section{Baseline Characteristics}

In total, 35 eyes of 35 participants were analyzed. All 35 participants ( 17 males and 18 females) completed this 12-month study. The ages of these participants ranged from 8 to 18 years $(12.7 \pm 3.0$ years old). The baseline cycloplegic spherical equivalent refraction for the 35 tested eyes was $-2.95 \pm$ $0.84 \mathrm{D}$. The mean horizontal visible iris diameter value was $12.1 \pm 0.4 \mathrm{~mm}$.

Participants slept in their homes and traveled to the hospital as soon as possible after awakening and lens removal. The mean duration between lens removal in the morning and the study measurements was $1.9 \pm 0.7$ hours for all the follow-up visits.

\section{Repeatability and Reproducibility}

The repeatability and reproducibility of the method used in this study are shown in Figure 2 (taking the Type I lens decentration vector as an example). The Bland-Altman figure was shown in Figure 2A-D (taking the figures of lens decentration and asymmetry vector between the observers as example). The standard deviation and coefficient of repeatability of three measurements by observers 1 and 2 was shown in Figure 2E, respectively. For either the lens decentration vector or corneal asymmetry vector, Cronbach's alpha coefficient and the intraclass correlation coefficient for each observer and between the observers were both greater than 0.95 . Therefore, the use of such a location and calculation method had acceptable repeatability and reproducibility.

\section{Lens Decentration}

\section{Magnitude and distribution.}


The overview of the two types of lens decentration over time is shown in Figure. 3. Concerning both the absolute decentration magnitude and the decomposed vectors, there were no significant differences between the two types of lens decentration (paired t-test, all p>.05). Only the data for Type I lens decentration was analyzed in further analyses.

The lens decentration magnitude (Figure 3A) (RM-ANOVA, post hoc, 1 month versus 1 week, $p=.032$ ), horizontal vector (Figure 3B) (RM-ANOVA, post hoc, 1 month versus 1 week, $p=.029$ ) and vertical vector (Figure 3C) (RM-ANOVA, post hoc, 1 month versus 1 week, $p=.045$ ) showed a significance decrease by 1 month. There were no significant changes throughout the rest of the study period. These data indicated that lens decentration tended to stabilize after 1 month.

We used the average lens decentration vectors after 1 month to represent the overall stable position, and these vectors were calculated by vector synthesis using all decomposed data from one month to one year to indicate the general decentration situation after stabilization. An overview of the averaged lens decentration for all the participants is presented in Figure 4A.

The magnitude of the averaged decentration varied from $0.07 \mathrm{~mm}$ to $1.35 \mathrm{~mm}(0.56 \pm 0.31 \mathrm{~mm})$. Most of the participants' lenses decentered by less than $1.0 \mathrm{~mm}(91 \%)$. In general, inferotemporal decentration was the most common (66\%). The mean angle of the averaged lens decentration was $203.2 \pm 49.2$ degrees.

\section{The corneal elevation asymmetry vector contributed to the averaged lens decentration}

The pretreatment corneal elevation asymmetry vector for all the participants is illustrated in Figure 4B. Linear regression analysis showed that the horizontal corneal elevation asymmetry vector significantly contributed to the averaged lens decentration along the horizontal coordinate $(R=0.374$, $\mathrm{p}=.027$ ) (Figure $5 \mathrm{~A}$ ), while the vertical asymmetry vector was nearly significantly correlated with the vertical average lens decentration vector $(R=0.327, p=.055)$ (Figure $5 B)$.

The mean angle of the corneal elevation asymmetry vector was $170.9 \pm 64.4$ degrees. It significantly contributed to the average lens decentration angle $(R=0.344, p=.043)$ (Figure 5C).

Otherwise, corneal astigmatism, $\mathrm{Q}$ values (including the mean and four quadrant values), $\mathrm{K}$ readings, the horizontal visible iris diameter and spherical equivalent refraction were not correlated with the average orthokeratology lens decentration vector in either the horizontal direction or the vertical direction, nor with its magnitude or angle (Linear regression analysis, all $p$ >.05).

\section{Discussion}


Our study results show that most of the lenses were decentered by less than $1.0 \mathrm{~mm}$. The cornea was healthy, and the uncorrected visual acuity was satisfactory. Hence, the lens decentration was tolerable and accepted. This result agrees with a previous study. ${ }^{22}$ An interesting finding is that orthokeratology lens decentration tended to stablize after the first month. We completed follow-up examinations for each participant for up to one year. It was verified that the location of lens decentration achieved high similarity after the first month, which was in agreement with the findings of a previous study. ${ }^{12}$ One possible explanation might be that the cornea needs time to reshape after lens wearing. ${ }^{23}$ The lens could be easily decentered before steady shaping. Just as glycosylated hemoglobin can be used to evaluate the average blood glucose level over the last three months, the average lens decentration vector could be applied to evaluate the general decentration after stabilization. Regarding the average lens decentration coordinate, the inferotemporal quadrant was the preferential orientation (66\%), which was consistent with the findings of previous studies. ${ }^{10,18}$

However, there is no generally accepted corneal factor that is believed to be correlated with lens decentration. Researchers have speculated that the lens decentration magnitude might be caused by nasal-temporal and superior-inferior corneal asymmetry, ${ }^{14}$ high degrees of corneal toricity ${ }^{13}$ and $\mathrm{Q}$ values at different meridians. ${ }^{15}$ Even ethnicity is a concern because Asian patients are believed to have much tighter eyelids, which is thought to cause a greater lens decentration magnitude compared to other ethnic groups (Tahhan, et al. IOVS 2003;44:ARVO E-Abstract 3714). An interesting result of this study was that lens decentration showed no relationship with corneal astigmatism or Q values at different meridians, which was in contrast to the findings reported in previous literature. ${ }^{13,15}$ This might be because only participants with refractive astigmatism of less than - 1.5 D were recruited in this study. The influence of corneal astigmatism and asphericity may be minimized in low astigmats, which is in agreement with the findings of Chen et al. ${ }^{14}$ Additionally, the current study found that lens decentration was independent of the corneal $\mathrm{K}$ readings, horizontal visible iris diameter and spherical equivalent refraction as long as the lens parameters was customized to the patient's corneal curvature, size and refractive condition.

In contrast, elevation data are much more straightforward than curvature data when used to estimate lens decentration based on the assumption that lenses tend to tilt toward the lower area of the anterior cornea. ${ }^{6}$ Our study illustrated that the corneal elevation asymmetry vector at the boundary between the reverse curve and the alignment curve was the only parameter of the cornea that significantly contributed to the average lens decentration. Researchers have found that the peripheral cornea is the main asymmetric area of the whole cornea, and the alignment curve of the orthokeratology lens is considered to play an important role in lens stabilization on the cornea. While the typical chord of the alignment curve of an orthokeratology lens design most likely falls between the chords of 7.0 and $10.0 \mathrm{~mm}$ (e.g., for the Euclid Emerald lens design, the alignment curve within the total lens diameter is between the chords of 7.20 and $9.60 \mathrm{~mm}), 6,24,25$ it is reasonable to consider that the sagittal height difference at the boundary between the reverse curve and the alignment curve is critical for lens centration. 
A previous study ${ }^{14}$ used corneal elevation data collected at the $8 \mathrm{~mm}$ chord of two respective principal meridians for corneal astigmatism to estimate the asymmetry of the cornea, which assumed the cornea as a typical toric surface. However, most corneas are not standardized toric surfaces. In our study, a customized program was designed to calculate the height difference of the anterior corneal elevation map at the chord of $7.2 \mathrm{~mm}$ (the boundary between the reverse curve and the alignment curve). The sum of the asymmetry vectors was 3 -dimensional, including all 256 semimeridians from $0^{\circ}$ to $360^{\circ}$. This result was present along both the horizontal and vertical axes, making it much more comprehensive and objective (Table 1).

Table 1

Repeatability and reproducibility of orthokeratology lens decentration location and asymmetry vector calculation

\begin{tabular}{|c|c|c|c|c|c|c|}
\hline & & & $\begin{array}{l}\text { SD } \\
(\mathrm{mm})\end{array}$ & $\begin{array}{l}\text { COR } \\
\text { (\%) }\end{array}$ & $\begin{array}{l}\text { Cronbach's } \\
\text { alpha }\end{array}$ & ICC (95\% Cl) \\
\hline \multirow[t]{3}{*}{$\begin{array}{l}\text { LD } \\
\text { (horizontal) }\end{array}$} & \multirow[t]{2}{*}{$\begin{array}{l}\text { For each } \\
\text { observer }\end{array}$} & $\begin{array}{l}\text { Observer } \\
1\end{array}$ & $\begin{array}{l}0.02 \pm \\
0.02\end{array}$ & 7.79 & 0.995 & $\begin{array}{l}0.988(0.981- \\
0.995)\end{array}$ \\
\hline & & $\begin{array}{l}\text { Observer } \\
2\end{array}$ & $\begin{array}{l}0.02 \pm \\
0.01\end{array}$ & 7.39 & 0.996 & $\begin{array}{l}0.988(0.981- \\
0.994)\end{array}$ \\
\hline & $\begin{array}{l}\text { Between } \\
\text { observers }\end{array}$ & & $\begin{array}{l}0.02 \pm \\
0.02\end{array}$ & 7.12 & 0.994 & $\begin{array}{l}0.994(0.988- \\
0.996)\end{array}$ \\
\hline \multirow[t]{3}{*}{$\begin{array}{l}\text { LD } \\
\text { (vertical) }\end{array}$} & \multirow[t]{2}{*}{$\begin{array}{l}\text { For each } \\
\text { observer }\end{array}$} & $\begin{array}{l}\text { Observer } \\
1\end{array}$ & $\begin{array}{l}0.03 \pm \\
0.02\end{array}$ & 7.81 & 0.996 & $\begin{array}{l}0.987(0.980- \\
0.993)\end{array}$ \\
\hline & & $\begin{array}{l}\text { Observer } \\
2\end{array}$ & $\begin{array}{l}0.03 \pm \\
0.03\end{array}$ & 7.64 & 0.996 & $\begin{array}{l}0.986(0.981- \\
0.994)\end{array}$ \\
\hline & $\begin{array}{l}\text { Between } \\
\text { observers }\end{array}$ & & $\begin{array}{l}0.02 \pm \\
0.02\end{array}$ & 7.06 & 0.993 & $\begin{array}{l}0.993(0.988- \\
0.995)\end{array}$ \\
\hline \multirow[t]{3}{*}{$\begin{array}{l}\text { Asymmetry } \\
\text { Vx }\end{array}$} & \multirow[t]{2}{*}{$\begin{array}{l}\text { For each } \\
\text { observer }\end{array}$} & $\begin{array}{l}\text { Observer } \\
1\end{array}$ & $\begin{array}{l}0.03 \pm \\
0.01\end{array}$ & 7.67 & 0.997 & $\begin{array}{l}0.988(0.981- \\
0.995)\end{array}$ \\
\hline & & $\begin{array}{l}\text { Observer } \\
2\end{array}$ & $\begin{array}{l}0.03 \pm \\
0.02\end{array}$ & 7.94 & 0.996 & $\begin{array}{l}0.988(0.981- \\
0.994)\end{array}$ \\
\hline & $\begin{array}{l}\text { Between } \\
\text { observers }\end{array}$ & & $\begin{array}{l}0.03 \pm \\
0.02\end{array}$ & 7.64 & 0.996 & $\begin{array}{l}0.994(0.988- \\
0.996)\end{array}$ \\
\hline \multirow[t]{3}{*}{$\begin{array}{l}\text { Asymmetry } \\
\text { Vy }\end{array}$} & \multirow[t]{2}{*}{$\begin{array}{l}\text { For each } \\
\text { observer }\end{array}$} & $\begin{array}{l}\text { Observer } \\
1\end{array}$ & $\begin{array}{l}0.02 \pm \\
0.03\end{array}$ & 7.46 & 0.996 & $\begin{array}{l}0.989(0.981- \\
0.994)\end{array}$ \\
\hline & & $\begin{array}{l}\text { Observer } \\
2\end{array}$ & $\begin{array}{l}0.02 \pm \\
0.02\end{array}$ & 7.39 & 0.996 & $\begin{array}{l}0.987(0.981- \\
0.994)\end{array}$ \\
\hline & $\begin{array}{l}\text { Between } \\
\text { observers }\end{array}$ & & $\begin{array}{l}0.01 \pm \\
0.02\end{array}$ & 7.39 & 0.994 & $\begin{array}{l}0.995(0.987- \\
0.996)\end{array}$ \\
\hline
\end{tabular}


A typical cornea with a higher corneal elevation asymmetry vector has a larger inferior than superior sagittal height and a larger temporal sagittal height than nasal sagittal height. Such asymmetry may cause the treatment zone to dislocate more inferiorly and temporally. Although linear regression analyses showed a significant correlation between the corneal elevation asymmetry vector and the averaged lens decentration vector in horizontal coordinates $(p=.027)$, only a nearly significant relationship was found between the asymmetry vector and the averaged lens decentration vector in the vertical direction ( $p$ $=.055)$. This might have been caused by other factors, such as the tension of the eyelids. External forces such as the eyelid force may aggravate the pushing of lenses inferiorly during overnight wear (Tahhan, et al. IOVS 2003;44:ARVO E-Abstract 3714). Of note is that the mean angle of the pretreatment corneal elevation asymmetry vector was superotemporal (170.9 \pm 64.4 degrees), while the mean angle of the averaged lens decentration was towards the inferotemporal direction (203.2 \pm 49.2 degrees). Although the two parameters were significantly correlated $(p=0.043)$, the eyelid force might have played a role in lens decentration in the vertical direction.

Another interesting question is whether the corneal vertex or the pupil center is a better reference position for the orthokeratology treatment center. ${ }^{26,27}$ The corneal vertex is approximately the center of the topographic map, which is based on the Placido principle. It is a highly useful reference for describing the geometric properties of the cornea. ${ }^{28,29}$ The pupil center is the representative center of the light channel. Orthokeratology retards myopia progression via the myopic defocusing of light. ${ }^{26}$ Therefore, which of these locations should be the attempted treatment center for orthokeratology is debatable. In this study, we used both the corneal vertex and the pupil center as reference points. Although the parameters of the lens decentration relative to different reference points were not significantly different, the Type I lens decentration parameters were smaller than those of Type II lens decentration. One explanation for this finding is that the purpose of lens fitting is to achieve ideal centration of the orthokeratology lens on the anterior corneal surface; hence, it may be more ideal to use Type I lens decentration parameters, rather than Type II.

One limitation of this study is that only baseline corneal parameters were sampled and applied to analyze possible correlations with lens decentration. Due to the limited methods, other potential factors, such as the position of the eyes during sleep, the tightness of the eyelids, the rapid eye movement phase, and the condition of the tear film, were not taken into consideration. ${ }^{30,31}$ This might be the reason why the $\mathrm{R}$ coefficients of linear regression were not large enough. Since Asian patients often have much tighter eyelids, the results of this study could be different from the results for other ethnic groups. In addition, the intervals between lens removal and the time at which the study measurements were taken differed. Based on current knowledge, we do not know how regression of the orthokeratology effect would influence the parameters of the treatment zone, specifically those used to define decentration.

In conclusion, the association between pretreatment corneal elevation asymmetry vector and one-yearaveraged orthokeratology lens decentration may assist in fitting and understanding the performance of orthokeratology lenses. In the future, this MATLAB program could be turned into a mature application, such as a topography software plugin. Based on this study, ophthalmologists and optometrists can use 
the pretreatment corneal asymmetry vector to partly predict lens decentration tendency in typical patients. But other factors, such as the tightness of the eyelids and so on also should be taken into consideration in clinical operation. Orthokeratology lens decentration is a common phenomenon that mostly occurs toward the inferior and temporal quadrants of the cornea in Chinese myopic patients. The lens decentration varied with time during early treatment and stabilized after the first month. To some extent, the one-year general state of lens decentration could be predicted based on the corneal elevation asymmetry vector, which is a novel parameter that can be calculated based on an elevation map.

\section{Declarations}

\section{Funding}

This work was funded by the National Natural Science Foundation of China (Grant No. 82000928 \& 81870684 \& 81770943 \& 81470651), the HuaXia Translational Medicine Fund for Young Scholars (Grant No. 2017-B-001), the Non-profit Central Research Institute Fund of Chinese Academy of Medical Sciences under a grant (Grant No. 2019HY320001), the National Key Instrumentation Development Project of China (Grant No. 2013YQ030651), the National key research and development program (Grant No. 2016YFC0904801), and the Research Fund for Science and Technology Program of Beijing (Grant No. Z161100000516037). The funders had no role in the study design, data collection and analysis, decision to publish or preparation of the manuscript.

\section{Conflicts of interest}

The authors declare that they have no conflicts of interest.

\section{Informed consent}

Informed consent was obtained from all individual participants included in the study.

\section{References}

1. Xie P, Guo X (2016) Chinese Experiences on Orthokeratology. Eye Contact Lens 42:43-47. https://doi.org/10.1097/ICL.0000000000000190

2. Cho P, Cheung SW (2012) Retardation of myopia in Orthokeratology (ROMIO) study: a 2-year randomized clinical trial. Invest Ophthalmol Vis Sci 53:7077-7085. https://doi.org/ 10.1167/iovs.1210565

3. Walline JJ, Jones LA, Sinnott LT (2009) Corneal reshaping and myopia progression. Br J Ophthalmol 93:1181-1185. https://doi.org/10.1136/bjo.2008.151365 
4. Kakita T, Hiraoka T, Oshika T (2011) Influence of overnight orthokeratology on axial elongation in childhood myopia. Invest Ophthalmol Vis Sci 52:2170-2174. https://doi.org/ 10.1167/iovs.10-5485

5. Charm J, Cho P (2013) High myopia-partial reduction ortho-k: a 2-year randomized study. Optom Vis Sci 90:530-539. https://doi.org/10.1097/OPX.0b013e318293657d

6. Swarbrick HA (2004) Orthokeratology (corneal refractive therapy): what is it and how does it work? Eye Contact Lens 30:181-185; discussion 205 - 186. https://doi.org/ 10.1097/01.icl.0000140221.41806.6e

7. Swarbrick HA, Alharbi A, Watt K et al (2015) Myopia control during orthokeratology lens wear in children using a novel study design. Ophthalmology 122:620-630. https://doi.org/ 10.1016/j.ophtha.2014.09.028

8. Sun Y, Wang L, Gao J et al (2017) Influence of Overnight Orthokeratology on Corneal Surface Shape and Optical Quality. J Ophthalmol 2017:3279821. https://doi.org/10.1155/2017/3279821

9. Liu G, Chen Z, Xue F et al (2018) Effects of Myopic Orthokeratology on Visual Performance and Optical Quality. Eye Contact Lens 44:316-321. https://doi.org/ 10.1097/ICL.0000000000000372

10. Hiraoka T, Mihashi T, Okamoto $C$ et al (2009) Influence of induced decentered orthokeratology lens on ocular higher-order wavefront aberrations and contrast sensitivity function. J Cataract Refract Surg 35:1918-1926. https://doi.org/ 10.1016/j.jcrs.2009.06.018

11. Hiraoka T, Okamoto F, Kaji Y et al (2006) Optical quality of the cornea after overnight orthokeratology. Cornea 25:S59-S63. https://doi.org/ 10.1097/01.ico.0000247215.08886.46

12. Jiang J, Lian L, Wang F et al (2019) Comparison of Toric and Spherical Orthokeratology Lenses in Patients with Astigmatism. J Ophthalmol 2019:4275269. https://doi.org/10.1155/2019/4275269

13. Maseedupally VK, Gifford P, Lum E et al (2016) Treatment Zone Decentration During Orthokeratology on Eyes with Corneal Toricity. Optom Vis Sci 93:1101-1111. https://doi.org/ 10.1097/OPX.0000000000000896

14. Chen Z, Xue F, Zhou J et al (2017) Prediction of Orthokeratology Lens Decentration with Corneal Elevation. Optom Vis Sci 94:903-907

15. Li J, Yang C, Xie W et al (2017) Predictive role of corneal Q-value differences between nasal-temporal and superior-inferior quadrants in orthokeratology lens decentration. Med (Baltim) 96:e5837. https://doi.org/10.1097/MD.0000000000005837

16. Dave T, Ruston D (1998) Current trends in modern orthokeratology. Ophthalmic Physiol Opt 18:224233

17. Paune J, Cardona G, Quevedo L (2012) Toric double tear reservoir contact lens in orthokeratology for astigmatism. Eye Contact Lens 38:245-251. https://doi.org/10.1097/ ICL.0b013e318258789e

18. Zhang Y, Chen YG (2018) Comparison of myopia control between toric and spherical periphery design orthokeratology in myopic children with moderate-to-high corneal astigmatism. Int $\mathrm{J}$ Ophthalmol 11:650-655. https://doi.org/ 10.18240/ijo.2018.04.19 
19. Cho P, Cheung SW, Mountford J et al (2008) Good clinical practice in orthokeratology. Cont Lens Anterior Eye 31:17-28. https://doi.org/10.1016/j.clae.2007.07.003

20. McAlinden C, Khadka J, Pesudovs K (2011) Statistical methods for conducting agreement (comparison of clinical tests) and precision (repeatability or reproducibility) studies in optometry and ophthalmology. Ophthalmic Physiol Opt 31:330-338. https://doi.org/ 10.1111/j.14751313.2011.00851.x

21. Bland MJA, Douglas G (1986) Statistical Methods for Assessing Agreement between Two Methods of Clinical Measurement. Lancet 1:931-936

22. Chen J, Huang W, Zhu R et al (2018) Influence of overnight orthokeratology lens fitting decentration on corneal topography reshaping. Eye Vis (Lond) 5:5. https://doi.org/ 10.1186/s40662-018-0100-7

23. Alharbi A, Swarbrick HA (2003) The effects of overnight orthokeratology lens wear on corneal thickness. Invest Ophthalmol Vis Sci 44:2518-2523. https://doi.org/ 10.1167/iovs.02-0680

24. Mika R, Morgan B, Cron M et al (2007) Safety and efficacy of overnight orthokeratology in myopic children. Optometry 78:225-231. https://doi.org/10.1016/j.optm.2006.12.013

25. Tahhan N, Du Toit R, Papas E et al (2003) Comparison of reverse-geometry lens designs for overnight orthokeratology. Optom Vis Sci 80:796-804. https://doi.org/ 10.1097/00006324-200312000-00009

26. Reinstein DZ, Archer TJ, Gobbe M (2012) Is topography-guided ablation profile centered on the corneal vertex better than wavefront-guided ablation profile centered on the entrance pupil? J Refract Surg 28:139-143. https://doi.org/10.3928/1081597X-20111115-01

27. Atchison DA (2006) Optical models for human myopic eyes. Vision Res 46:2236-2250. https://doi.org/10.1016/j.visres.2006.01.004

28. Read SA, Collins MJ, Carney LG et al (2006) The topography of the central and peripheral cornea. Invest Ophthalmol Vis Sci 47:1404-1415. https://doi.org/10.1167/iovs.05-1181

29. Gu T, Gong B, Lu D et al (2019) Influence of Corneal Topographic Parameters in the Decentration of Orthokeratology. Eye Contact Lens 45:372-376. https://doi.org/ 10.1097/ICL.0000000000000580

30. Li J, Dong P, Liu H (2018) Effect of Overnight Wear Orthokeratology Lenses on Corneal Shape and Tears. Eye Contact Lens 44:304-307. https://doi.org/10.1097/ICL.0000000000000357

31. Nieto-Bona A, Nombela-Palomo M, Felipe-Marquez G et al (2018) Tear Film Osmolarity in Response to Long-Term Orthokeratology Treatment. Eye Contact Lens 44:85-90. https://doi.org/ 10.1097/ICL.0000000000000347

\section{Figures}




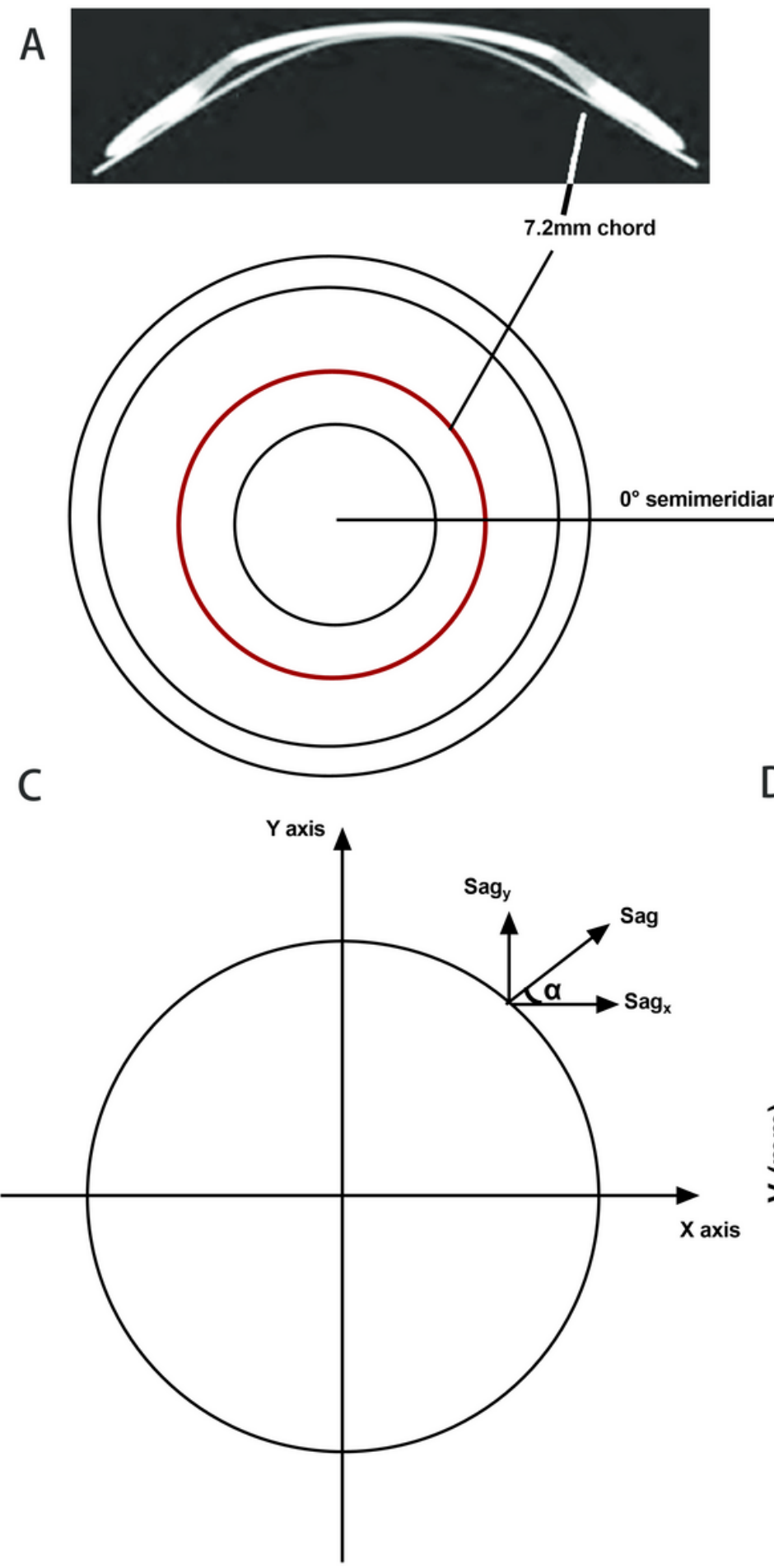

B

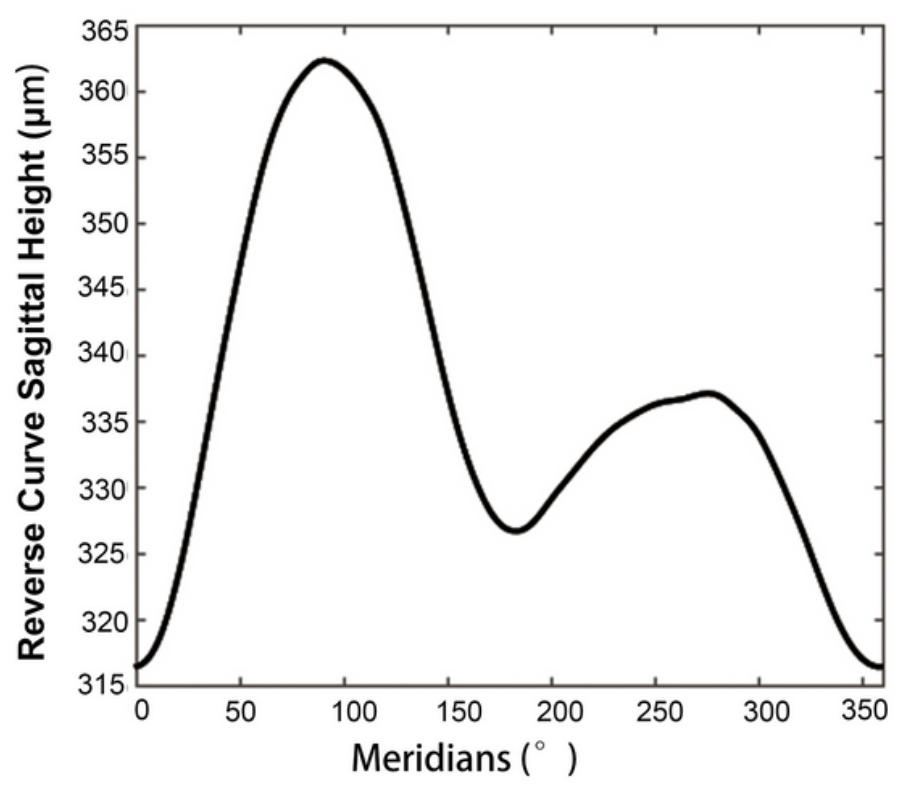

D

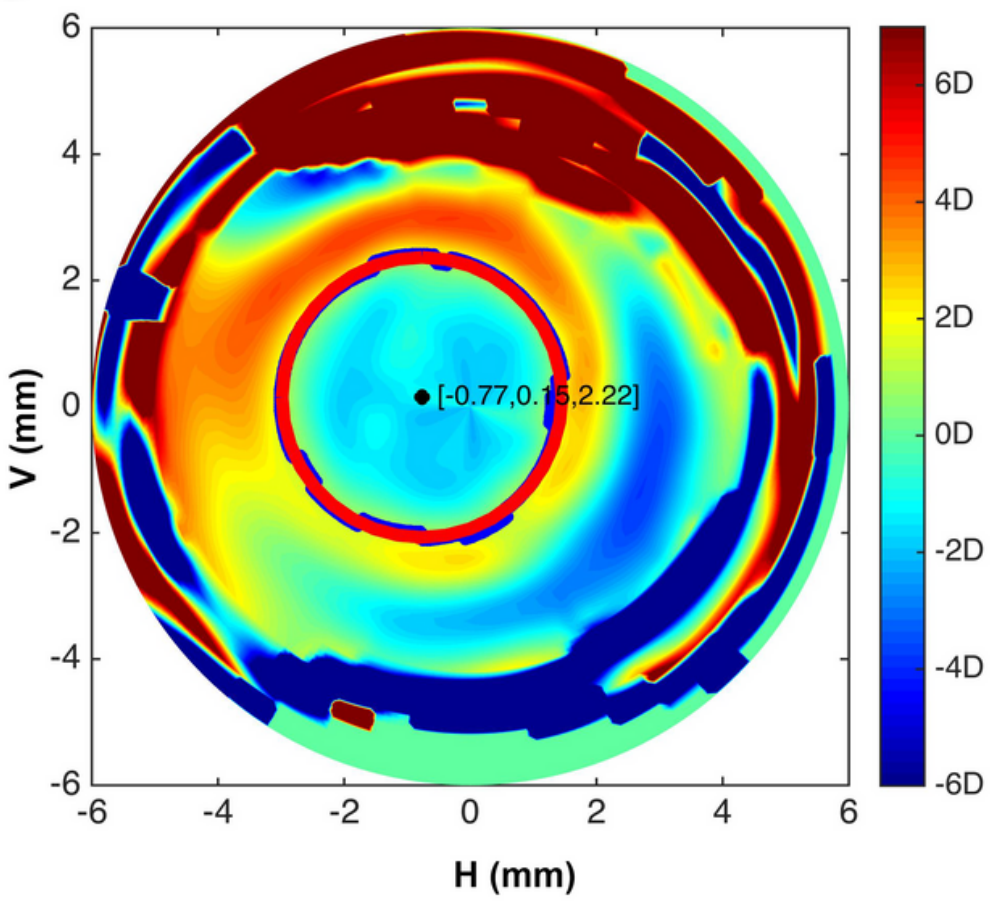

Figure 1

(A) The chord of $7.2 \mathrm{~mm}$. (B) Illustration of the sagittal height data of one patient, covering angles from $0^{\circ}-360^{\circ}$. The 256 data points are connected by a curved line. The sagittal height peaked at $90^{\circ}$ and $270^{\circ}$, suggesting a "with-the-rule" astigmatism. (C) Illustration of the sagittal height of one semimeridian. (D) A total of 256 blue points composes the border of the treatment zone. The red circle indicates the best-fit circle using the least-squares method. The unit applied to describe lens decentration on both the $x$-axis 
$(\mathrm{H})$ and $y$-axis $(\mathrm{V})$ is millimeters $(\mathrm{mm})$. In this figure, $-0.77 \mathrm{~mm}$ and $0.15 \mathrm{~mm}$ represent the decentration on the $x$ and $y$-axes, respectively, and $2.22 \mathrm{~mm}$ is the radius of the treatment zone.

A

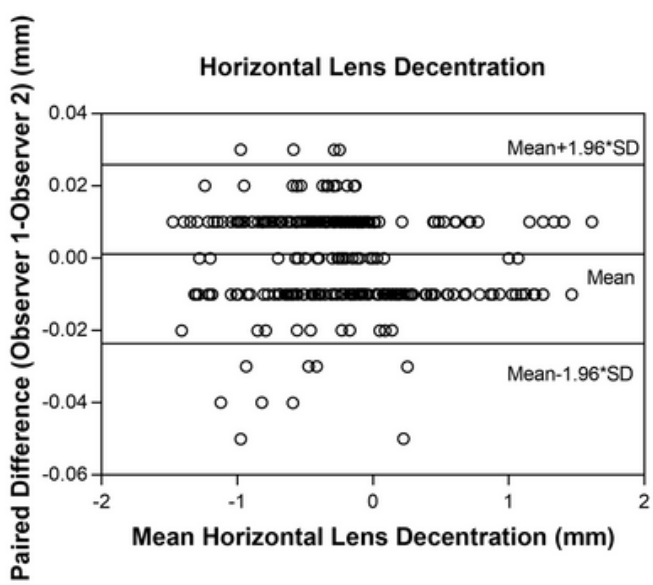

C

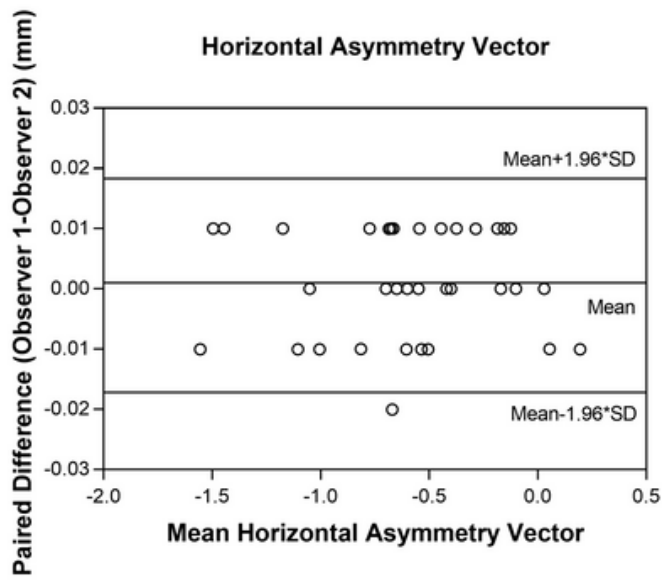

B
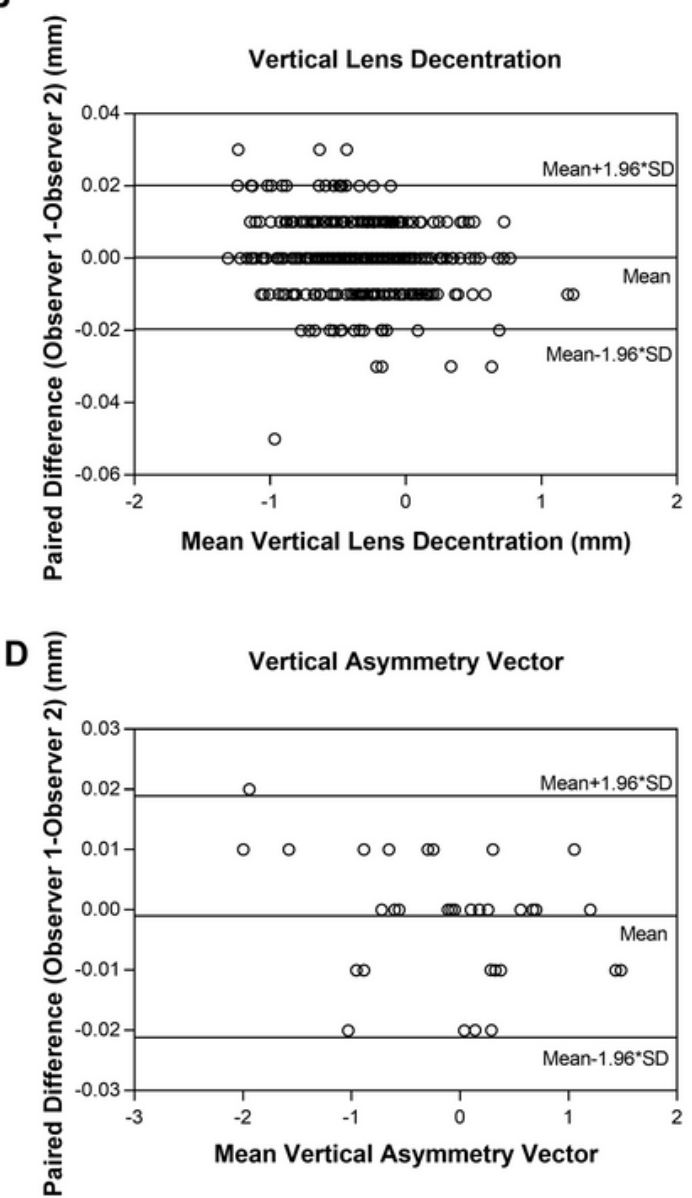

E

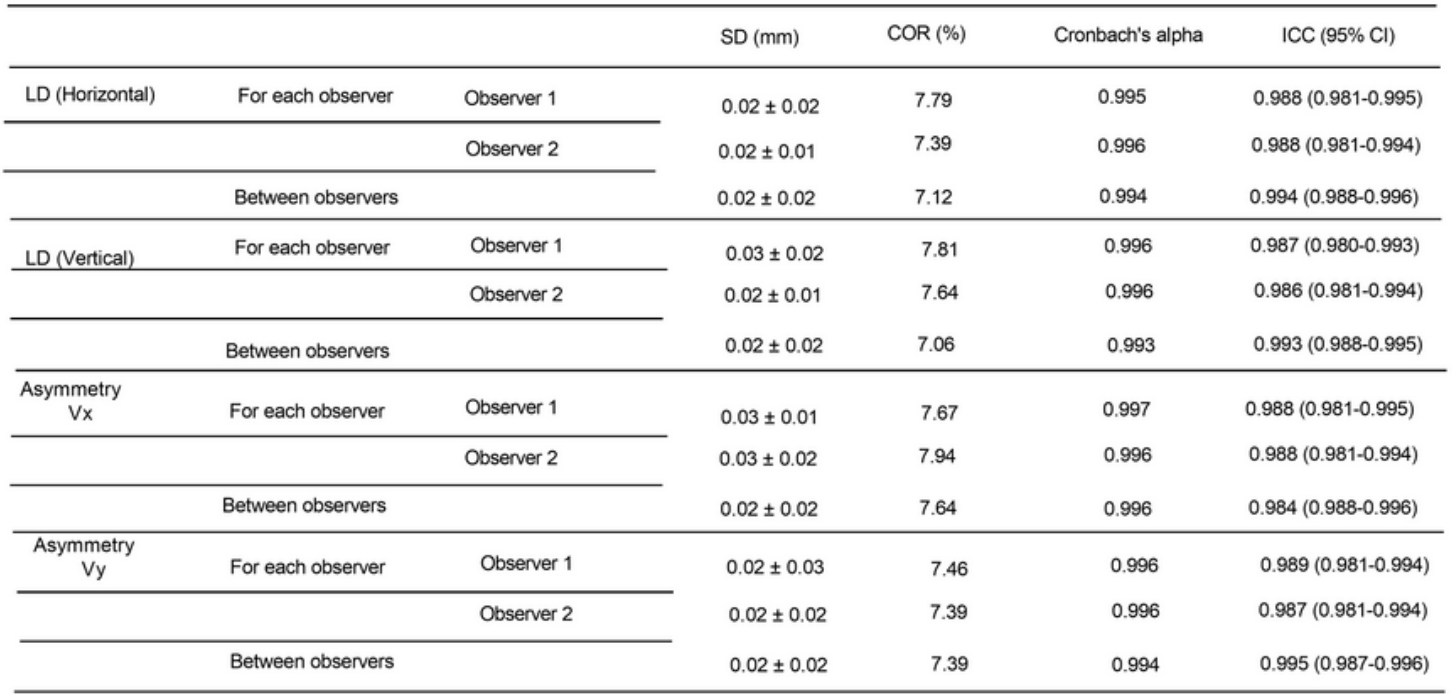

\section{Figure 2}

The repeatability and reproducibility of lens decentration and asymmetry vector measurements (taking the Type I lens decentration vector as an example). (A) The Bland-Altman figure of horizontal lens decentration between the observers. (B) The Bland-Altman figure of vertical lens decentration between the 
observers. (C) The Bland-Altman figure of horizontal asymmetry vector between the observers. (D) The Bland-Altman figure of vertical asymmetry vector between the observers. (E) The standard deviation, coefficient of repeatability, intraclass correlation coefficient of three measurements by observers 1 and 2 and between observers. * $\mathrm{LD}$, lens decentration; $\mathrm{SD}$, standard deviation; $\mathrm{COR}$, coefficient of repeatability; ICC, intraclass correlation coefficient.
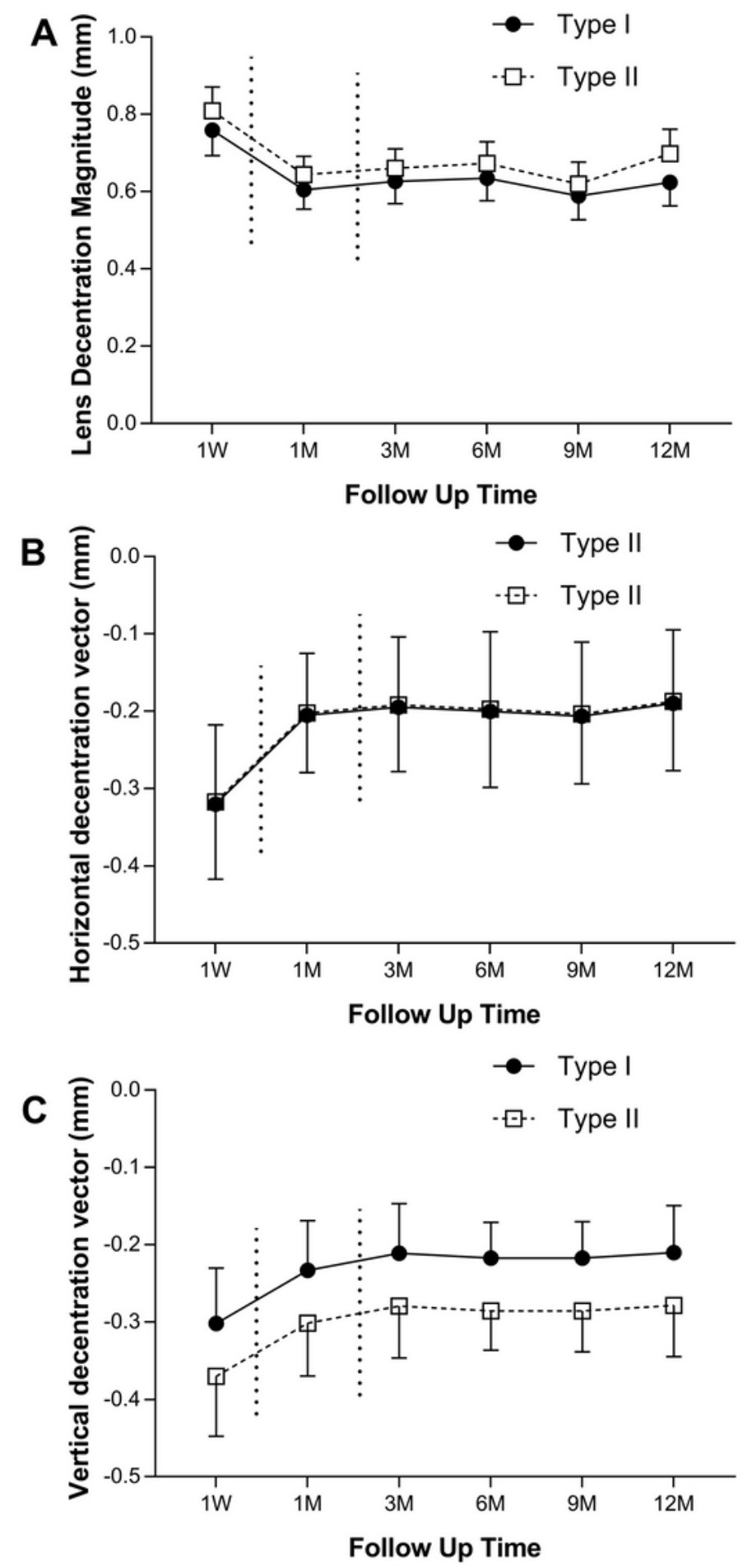

Figure 3 
Lens decentration magnitude (A) and its horizontal (B) and vertical (C) vectors at all visits over 12 months. The upper and lower error bars represent the SDs for Type I and Type II lens decentration, respectively. The dotted line after $1 \mathrm{w}$ and $1 \mathrm{~m}$ indicates non-continuous time interval. * $1 \mathrm{w}, 1$ week; $1 \mathrm{~m}, 1$ month; 3 m, 3 months; 6 m, 6 months; 9 m, 9 months; 12 m, 12 months.

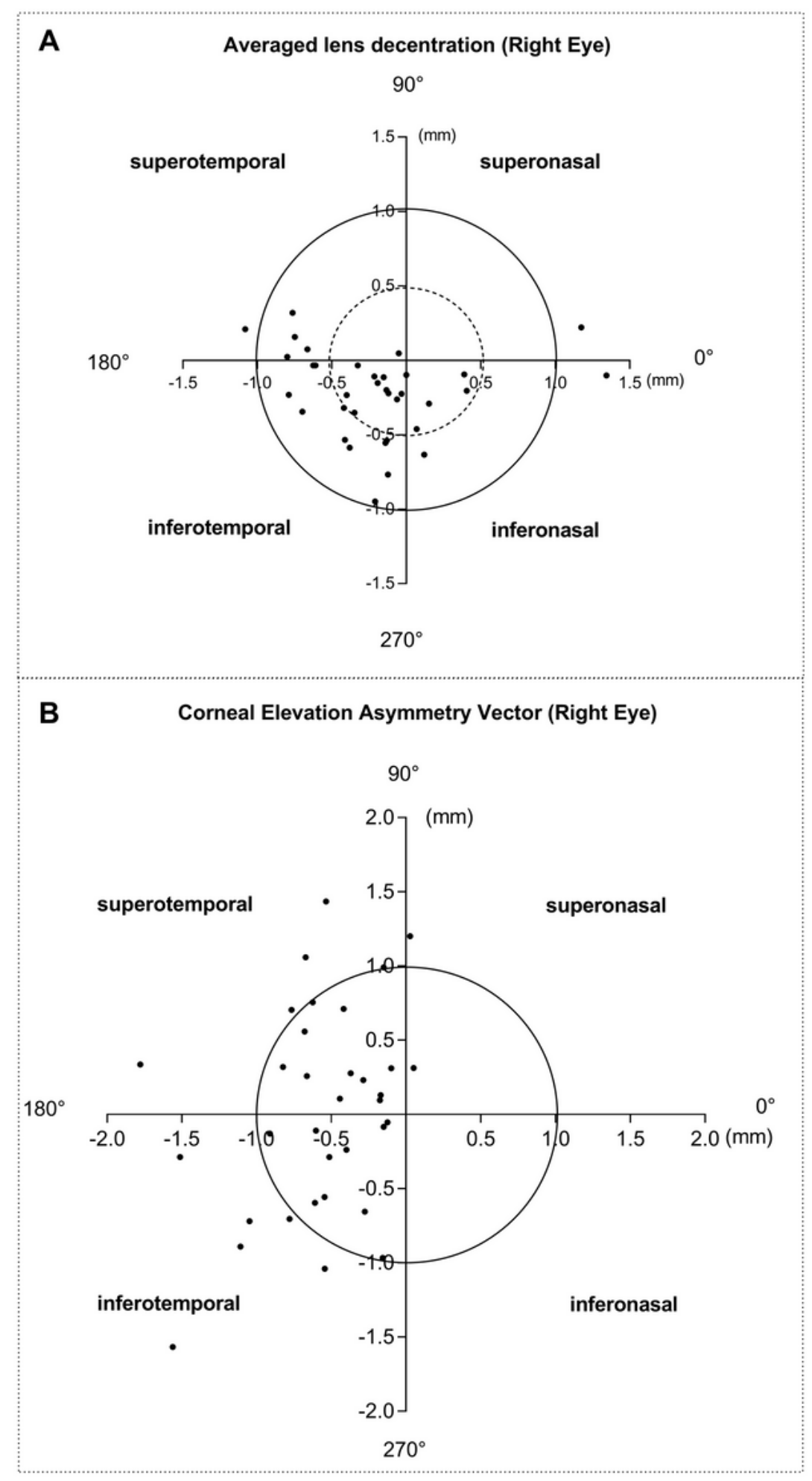

Figure 4 
Overview of the one-year-averaged lens decentration $(A)$ and pretreatment corneal elevation asymmetry vector $(B)$. The origin point of this coordinate system is the corneal vertex. The $0^{\circ}$ semimeridian starts from 3 o'clock on the topography map and moves counterclockwise to the $360^{\circ}$ semimeridian (back to 3 o'clock). Dashed and solid circles outline the distances of 0.5 and $1.0 \mathrm{~mm}$ to the corneal vertex.
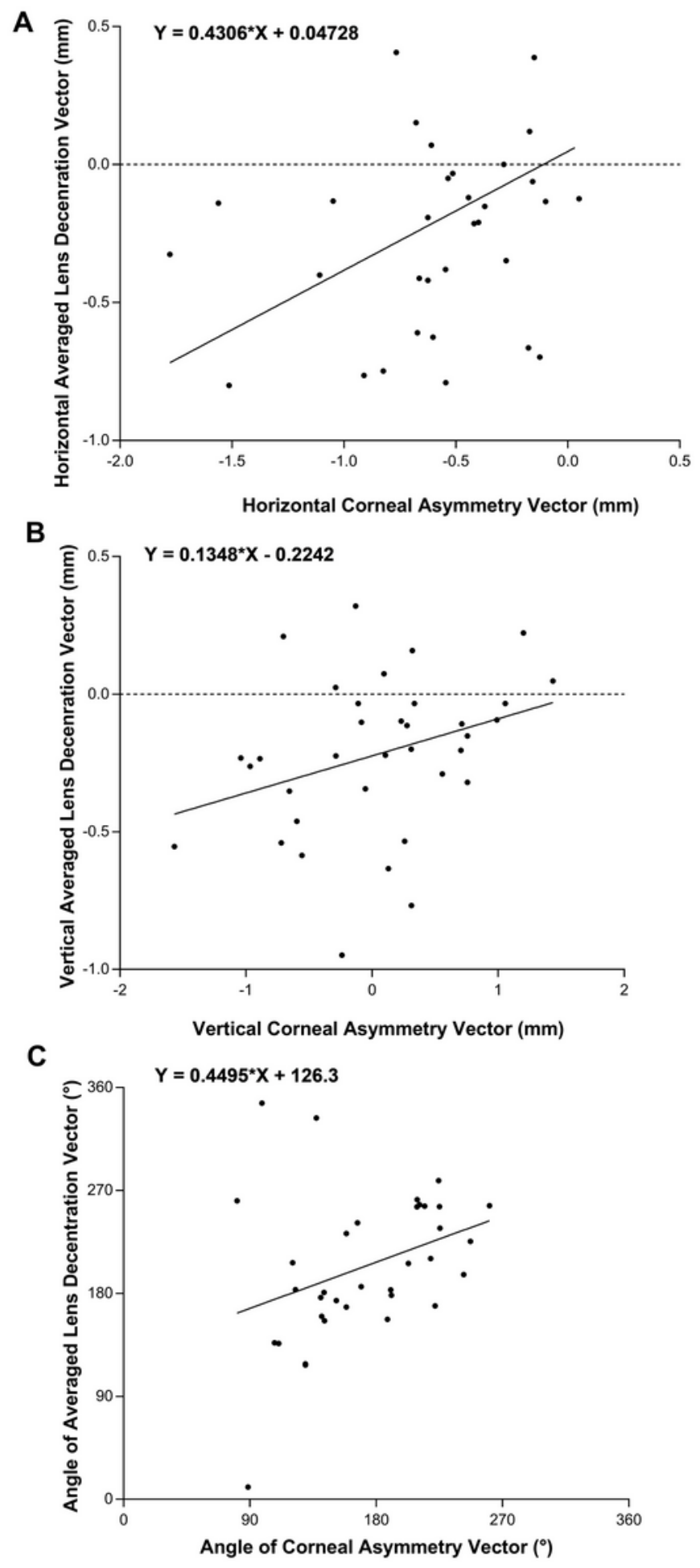

Figure 5 
Scatterplot showing that the pretreatment corneal elevation asymmetry vector and the average lens decentration vector were significantly correlated on the horizontal coordinate $(A)(R=0.374, p=.027)$ and nearly significantly correlated on the vertical coordinate $(B)(R=0.327, p=.055)$. Linear regression also showed that the corneal asymmetry vector angle and averaged lens decentration angle were statistically significantly correlated $(C)(R=0.344, p=.043)$. 